\title{
OSTEOMYELITIS OF MULTIPLE CRANIAL BONES - A RARE PRESENTATION
}

Elangovan $\mathrm{S}^{1}$, Srinivasa $\mathrm{V}^{2}$

\section{HOW TO CITE THIS ARTICLE:}

Elangovan S, Srinivasa V "Oste omyelitis of multiple cranial bones - A rare presentation". Journal of Evolution of Medical and Dental Sciences 2013; Vol. 2, Issue 43, October 28; Page: 8314-8317.

ABSTRACT: Background: Cases of osteomyelitis of maxilla were reported as a rare presentation. But osteomyelitis involving multiple cranial bones is a rarest of rare case. Here we present a case of 65 year old male, who presented with features of multiple discharging sinu ses intraorally, externally over left cheek and left post-auricular region. On evaluation, the condition was found to be chronic pyogenic osteomyelitis involving maxilla, zygoma and temporal bones and odontogenic in origin. AIM: We have decided to report this interesting case because of its extensive involvement of multiple cranial bones in this antibiotic era and complete resolution of infection was gained with antibiotic therapy and surgical treatment.

KEY WORDS: Osteomyelitis, Maxilla,Zygoma, Temporal bone.

INTRODUCTION: Osteomyelitis can be defined as an inflammatory condition of the bone, which begins as an infection of the medullary cavity, rapidly involves the haversian systems, a nd extends to involve the periosteum of the affected area. Infection occurs as a result of a bacteremia, an inoculation during aseptic surgery or bone surgery or a contiguous infectious focus. Conditions altering the vascularity of the bone such as radiation, malignancy, osteoporosis, osteopetrosis, and paget's disease predispose to osteomyelitis.1, 2 Systemic diseases like diabetes, anemia and malnutrition that cause concomitant alteration in host defenses profoundly influence the course of osteomyelitis. ${ }^{1,2}$

Dealing with osteomyelitis in head and neck bones is not the same as in other bones of the body due to the nature of the bones, complex anatomy of the region, and aesthetics. ${ }^{3}$ Treatment can be conservative resection of the diseased bone with adequate clearance in all cases along with appropriate antibiotic therapy. The general lack of awareness of the prevalence of the disease and its features often leads to a misdiagnosis and delay in treatment. Early detection of this condition and prompt attention will prevent the need for a surgical intervention in an otherwise protracted course of illness. 4,5

CASE REPORT: A 65-year-old male presented with discharging ulcer from left side upper part of the oral cavity for One and a half year, discharging sinus from left cheek-1yr, discharging sinus in post aural region-10 months.

Patient had undergone dental extraction 2yrs back from left upper set of teeth (2nd premolar and $1^{\text {st }}$ molar) for dental infection and 6 months later he noticed halitosis and purulent discharge in the oral cavity, 1yr back he noticed swelling in the left side of cheek which burst open and discharging yellowish pus, and leaving a sinus which persisted since then. 10 months back he started getting purulent discharge from left post aural region, copious in amount and foul smelling. There was no evidence of pulmonary tuberculosis. 


\section{CASE REPORT}

On general examination patient was moderately built, poorly nourished and anaemic. Systemic examination was within normal limit. On local examination poor orodental hygiene with multiple missing teeth in the left upper alveolus. The intra oral sinus of $1 \mathrm{x} 0.5 \mathrm{~cm}$ was present with whitish floor and was bony hard on digital palpation. (Fig.1) In the Left pre auricular area $2 \mathrm{~cm}$ in front of the left tragus showed hyperpigmentation of $1 \mathrm{x} 0.5 \mathrm{~cm}$ and with discharging sinus at the centre of this pigmentation. (Fig.2) Left post aural region showed a sinus. (Fig.3)There was purulent foul smelling discharge filling the external auditory canal and the posterior canal wall was eroded. Tympanic membrane was intact with grade- 1 retraction.

INVESTIGATIONS: Investigations revealed that the patient was anemic, non-diabetic and there was no evidence of pulmonary tuberculosis. Haemoglobin was $9.1 \mathrm{mg} / \mathrm{dl}$ and ESR was elevated. (1/2 hr$34 \mathrm{~mm}, 1 \mathrm{hr}-70 \mathrm{~mm}$ ). Gram staining and pus culture showed pseudomonas aeruginosa. CT scan showed osteomylelitic changes in maxilla, zygoma and temporal bones. (Fig. 4).

MANAGEMENT: Patient was administered cefotaxime and analgesics for one week. After culture and sensitivity report changed to ciprofloxacin. The discharge from the sinus and the pain were reduced. Patient was given surgical clearance of the diseased bone to ensure complete cure.

Approach - combined sublabial and postaural approach. Intra oral fistula tract traced by widening the area using microdrill. Bony sequestrum found in zygomatic process of maxilla and zygoma. External fistula tract of $2 \mathrm{cms}$ along with bits of bony sequestrae were removed. The wound closed after saucerization of the bone. Through post auricular approach bony sequestrum found in mastoid,squamous and zygomatic process of temporal bone and posterior wall of external auditory canal were removed. (fig-5) Specimen sent for histopathological examination. Report- Consistent with osteomyelitis.

DISCUSSION: Osteomyelitis is an acute or chronic inflammatory process that can involve cortical and trabecular aspects of bone or bone marrow. Osteomyelitis of the facial bones is a rare condition. Cranial bones are infrequently involved. Osteomyelitis of zygoma seldom occurs because the zygoma receives a relatively sufficient supply of blood. Furthermore, antibiotic therapy usually results in high rate of cure. A few cases of osteomyelitis of the facial bones have been reported and generally involve lesions of the maxilla or mandible that developed after trauma or dental infections. ${ }^{1,2}$

Osteomyelitis is an opportunistic infection that is usually a complication of some other condition rendering the host susceptible to disease. In tooth-bearing bone, osteomyelitis is usually caused by polymicrobial odontogenic bacteria. In long bones, classically Staphylococcus aureus is the offending microbe. Bacteroides, Peptostreptococcus, and microaerophilic Streptococcus spp., as well as other opportunistic pathogens, make up the odontogenic species that affect toothbearing bone. Other newer-found organisms include Arachnia, Klebsiella, mycobacterium tuberculosis, and Eikenella spp. Fungal organisms, such as Candida parapsilosis and Aspergillus, also have been reported causing craniofacial osteomyelitis especially in patients who are immunocompromised. 4,5

Chronic osteomyelitis may present like acute osteomyelitis with the inclusion of a chronic draining fistula. Chronic osteomyelitis is a very serious condition requiring immediate hospitalization followed by aggressive surgical and antibiotic therapy. In this disease, the blood 


\section{CASE REPORT}

supply to the infected area is usually severely compromised. Most authors agree that antibiotics should be continued much longer than usual for odontogenic infections. For chronic osteomyelitis, antibiotic treatment may be continued for up to 6 months. 3,5

In our case the patient was aged, poorly nourished and anaemic which could be the underlying causes. The pathogenic organism was pseudomonas aeruginosa which is unusual and treated earlier with inappropriate antibiotics along with delay in the diagnosis were the causes for extension of osteomyelitis to multiple cranial bones.

\section{REFERENCES:}

1. Kishore Chandra Prasad, Sampath Chandra Prasad, Neela Mouli \& Salil Agarwal Acta OtoLaryngologica, 2007;127: 194-205.

2. Topazian RG. Osteomyelitis of jaws. In: Topazian RG, Goldberg MH, editors. Oral and maxillofacial infections, 3rd edn. Philadelphia, PA: Saunders; 1994. p. 251-86.

3. Prasad.S,Isser.D.K, Osteomyelitis of the Maxilla, Indian journal of Otolaryngology 1990; vol 42,No. 1:41-42.

4. Indrizzi E, Terenzi V, Renzi G, Bonamini M, Bartolazzi A, Fini G. Journal of Craniofacial Surg. 2005 Sep;16(5):861-4.

5. David J. Pincus, M.D., ${ }^{1}$ Milton B. Armstrong, M.D., ${ }^{1}$ and Seth R. Thaller, M.D., Semin Plast Surg. 2009 May; 23(2): 73-79.

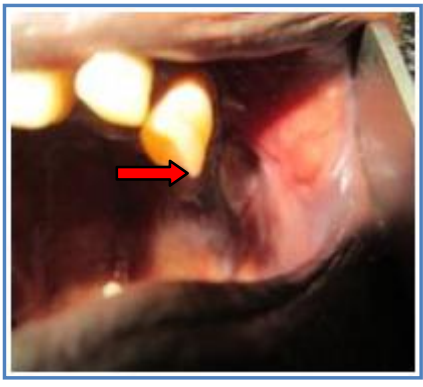

Intra-oral Fistula

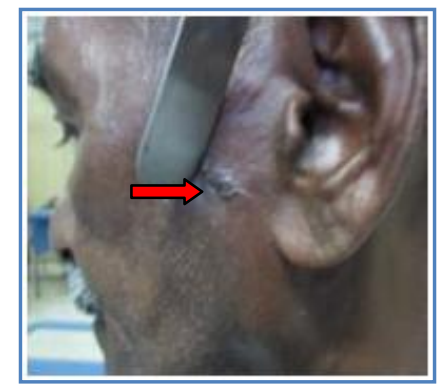

Fistula in the left cheek

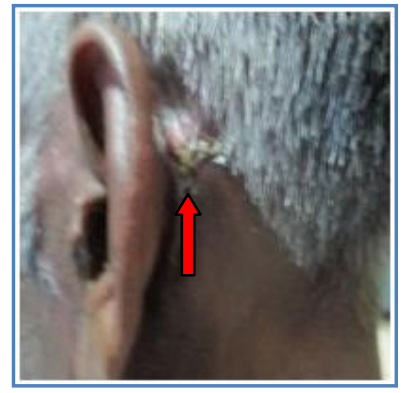

Fistula in the post-auricular region

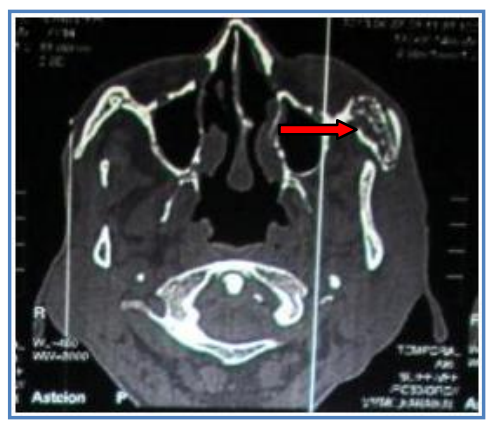

CT scan showing osteomyelitis of zygoma

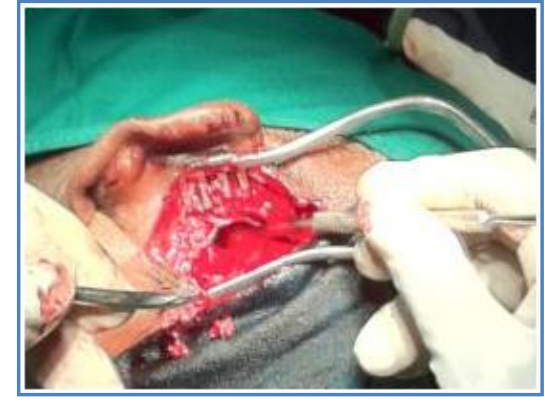

Removal of sequestrum from mastoid 


\section{AUTHORS:}

1. Elangovan $\mathrm{S}$.

2. Srinivasa V.

\section{PARTICULARS OF CONTRIBUTORS:}

1. Professor, Department of ENT, Vinayaka Mission's Medical College \& Hos pital, Karaikal, Puducherry-UT.

2. Professor \& HOD, Department of ENT, Vinayaka Mission's Medical College \& Hospital, Karaikal, Puducherry-UT.

\section{NAME ADDRESS EMAIL ID OF THE} CORRESPONDING AUTHOR:

Dr. Elangovan. S,

24, Golden Nagar,

P.K. Salai, Karaikal, PIN - 609602,

Puducherry-U T.

Email - hairikshini@yahoo.co.in

Date of Submission: 14/10/2013.

Date of Peer Review: 15/10/2013.

Date of Acceptance: 22/10/2013.

Date of Publishing: 24/10/2013 